\title{
THE MODULAR GROUP-RING OF A FINITE $p$-GROUP
}

\author{
D. L. JOHNSON ${ }^{1}$
}

\begin{abstract}
For a finite $p$-group $G$ and the field $k$ of $p$ elements, we investigate the embedding of $G$ in the group $G^{*}$ of elements of the groupring $k G$ having coefficient-sum equal to 1 . Of particular interest is the question of when $G$ has a normal complement in $G^{*}$, for in this case simple proofs can be given for a number of diverse known results.
\end{abstract}

Since its inception in [5], the study of modular group-rings of finite $p$-groups has largely centred around the problem (see [1], [3], for example): "When does isomorphism of group-rings imply isomorphism of groups?" A key role in these investigations has been played by the group of units in the group-ring.

Fixing our notation, we let $p$ denote a fixed prime, $G$ a finite $p$-group, $k$ the field of $p$ elements and $k G$ the group-ring of $G$ over $k$. The group of units of $k G$ is simply $k G \backslash U$, where $U$ is the augmentation ideal of $k G$. Furthermore, $k G \backslash U=G^{*} \times k^{*}$, where $G^{*}=1+U$, and $k^{*}=k \backslash\{0\}$. We call $G^{*}$ the mod $p$ envelope of $G$, and denote the embedding $G \succ G^{*}$ by $\iota_{G}$, or simply $\iota$. Note that $G^{*}$ is also a $p$-group.

Some properties of $\iota$ are as follows:

$$
\begin{aligned}
\mathbf{Z}(G) & =\mathbf{Z}\left(G^{*}\right) \cap G, \\
\mathbf{N}_{G^{*}}(G) & =G \mathbf{C}_{G^{*}}(G), \\
G^{\prime} & =\left(G^{*}\right)^{\prime} \cap G, \\
\Phi(G) & =\Phi\left(G^{*}\right) \cap G .
\end{aligned}
$$

None of these is very hard; (2) and (3) are proved in [2], and (4) in [6]. We have not yet, however, found a proof for

$$
G^{p}=\left(G^{*}\right)^{p} \cap G
$$

Incidently, the truth of (2) was established independently by the present author in an (entirely abortive) attempt to find a simple proof of Gaschutz' theorem [4].

We now define the class $\mathscr{L}_{p}$ of finite $p$-groups to consist of those $G$ which have a normal complement in $G^{*}$. The properties (1)-(5), along with many others, are immediately obvious for groups in $\mathfrak{L}_{p}$, and it only remains to

Received by the editors October 18, 1973.

AMS (MOS) subject classifications (1970). Primary 20D15; Secondary 16A26, $20 \mathrm{C05}$.

${ }^{1}$ The author is grateful to L. E. Moran and R. Tench for their kind permission to include the results embodied in Theorems 2, 4 and 7. 
establish the extent of this class. The purpose of this article is to outline the present state of our knowledge about this problem.

The embedding $\iota: G \gg G^{*}$ gives rise to a number of other questions which are beyond the scope of this article. For instance, what (if anything) can be said about the direct limit of the sequence $G \subseteq G^{*} \subseteq G^{* *} \subseteq \cdots$ ? Can the embedding $G \subseteq G^{*}$ be defined by any useful universal property? Are there any significant differences in the theory when $k$ is replaced by an arbitrary field of characteristic $p$ ? Can we find a formula relating $\exp G$ and $\exp G^{*}$ ? While these are equal for abelian groups, equality does not hold in general, as the example of the elementary nonabelian group of order $p^{3}$ shows (see [6]).

It would also be of interest to investigate the relations between the modular representation theory (and also the cohomology) of $G$ and $G^{*}$, for if $E(G)$ denotes the representation algebra of $\dot{G}$ (the vector space over the complex numbers with isomorphism classes of indecomposable $k G$-modules as a basis, and multiplication the Kronecker product of representations), then the definition of $G^{*}$ ensures that the restriction homomorphism: $E\left(G^{*}\right) \rightarrow E(G)$ splits.

Finally, and perhaps hardest of all, is there an algorithm for finding a presentation for $G^{*}$ (in terms of generators and relations) given a presentation of $G$ ?

THEOREM 1. If $G$ is cyclic, then $G \in \mathcal{L}_{p}$.

Proof. There is an elementary result, which may be thought of as a lemma for the basis theorem or as a consequence of it, to the effect that for any finite abelian $p$-group $G$ and any $x \in G$ with $|x|=\exp G,\langle x\rangle$ is a direct factor of $G$. This, together with the obvious remark that $\exp G=\exp G^{*}$ when $G$ is abelian, proves the result.

We next give a simpler proof of a result in [6].

TheOrem 2. If $G, H \in \mathcal{L}_{p}$, then $G \times H \in \mathcal{L}_{p}$.

Proof. The epimorphisms from $G \times H$ to $G$ and $H$ induce epimorphisms

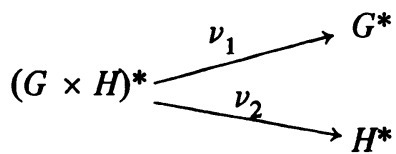

If $N_{1}$ and $N_{2}$ are normal complements (which exist by hypothesis) for $G$ and $H$ in $G^{*}$ and $H^{*}$, respectively, then let $\bar{N}_{1}$ and $\bar{N}_{2}$ denote their pre-images in $(G \times H)^{*}$ under $\nu_{1}$ and $\nu_{2}$ respectively. $\bar{N}_{1}$ and $\bar{N}_{2}$ are clearly normal subgroups of $(G \times H)^{*}$ such that

$$
\bar{N}_{1} \cap(G \times H)=H, \quad \bar{N}_{2} \cap(G \times H)=G,
$$

so that if we let $N=\bar{N}_{1} \cap \bar{N}_{2}$, then $N \cap(G \times H)=E$. Furthermore, 


$$
\begin{aligned}
\left|(G \times H)^{*}: N\right| & \leqslant\left|(G \times H)^{*}: \bar{N}_{1}\right|\left|(G \times H)^{*}: \bar{N}_{2}\right| \\
& =\left|G^{*}: N_{1}\right| H^{*}: N_{2}|=| G|| H|=| G \times H \mid .
\end{aligned}
$$

Thus $N$ is the required normal complement.

TheOREM 3. If $G$ is abelian, then $G \in \mathcal{L}_{p}$.

Proof. An immdiate consequence of Theorems 1 and 2.

The next result is due to Tench [8], and yields the converse of Theorem 2.

THEOREM 4. If $G$ belongs to $\mathcal{L}_{p}$, then so does any normally complemented subgroup $H$ of $G$.

Proof. Let $\alpha: H^{*} \rightarrow G^{*}$ be the inclusion induced by $H \leqslant G$, let $\beta$ : $G^{*} \rightarrow G$ be a splitting for $\iota_{G}$, and let $\gamma: G \rightarrow H$ be a splitting for $H \leqslant G$. Then the composite

$$
H^{*} \stackrel{\alpha}{\rightarrow} G^{*} \stackrel{\beta}{\rightarrow} G \stackrel{\gamma}{\rightarrow} H
$$

is clearly a splitting for $\iota_{H}$, whose kernel is thus the required normal complement.

THEOREM 5. For any $G, G^{*} \in \mathcal{L}_{p}$.

Proof. Note that $G^{*}$, being a subset of $k G$, is closed under the formation of linear combinations of its elements, provided the coefficient-sum is equal to 1 . Now any element of $G^{* *}$ is just a "formal" linear combination of this type, and thus gives rise to a unique element of $G^{*}$ (the corresponding "real" linear combination). This mapping is easily seen to be an epimorphism from $G^{* *}$ to $G^{*}$ which fixes $G^{*}$ elementwise. It is thus a splitting for $\iota_{G^{*}}$ and its kernel is the required normal complement.

THEOREM 6. If $G_{n}$ denotes the Sylow p-subgroup of $G L(n, p)$, then $G_{n} \in \mathcal{L}_{p}$ for all $n$.

Proof. The embedding $G_{n} \rightarrow G L(n, p) \rightarrow M_{n}(k)$ extends by linearity to a homomorphism of rings $k G_{n} \rightarrow M_{n}(k)$ whose restriction to $G_{n}^{*}$ is such that all its images are units in $M_{n}(k)$. We thus obtain a homomorphism $\alpha: G_{n}^{*} \rightarrow$ $G L(n, p)$ which fixes $G_{n}$ elementwise. But since $G_{n}^{*}$ is a $p$-group and $G_{n}$ is a Sylow $p$-subgroup of $G L(n, p)$, we must have $\operatorname{Im} \alpha=G_{n}$, so that $\operatorname{Ker} \alpha$ forms the required normal complement.

Note. By examining certain sets of upper triangular matrices with l's on the main diagonal, this argument can be extended to show that various other $p$-subgroups of $G L(n, p)$ lie in $\mathcal{L}_{p}$. Note further that if we knew $\mathcal{L}_{p}$ to be subgroup-closed, it would follow from Theorem 6 (or from Theorem 5) that $\mathcal{L}_{p}$ contained all finite $p$-groups.

Finally, for the sake of completeness, we list a few groups of small order in $\mathcal{L}_{p}$ (see [7] for the proof).

THEOREM 7. The following groups belong to $\mathcal{L}_{p}$ : 
(a) the two nonabelian groups of order $8(p=2)$,

(b) the nonabelian group of exponent $p$ and order $p^{3}(p>2)$,

(c) the three nonabelian indecomposable groups of exponent 4 and order 16 $(p=2)$.

Note in conclusion that the "smallest" group not definitely known to belong to $e_{p}$ is the dihedral group $D_{16}$ of order $16(p=2)$. A programme involving the conjugacy classes of $D_{16}^{*}$ is currently in preparation to decide the question using a high-speed computing machine.

\section{REFERENCES}

1. D. B. Coleman, Finite groups with isomorphic group algebras, Trans. Amer. Math. Soc. 105 (1962), 1-8. MR 26 \# 191.

2. On the modular group ring of a p-group, Proc. Amer. Math. Soc. 15 (1964), 511-514. MR 29 \#2306.

3. W. E. Deskins, Finite Abelian groups with isomorphic group algebras, Duke Math. J. 23 (1956), 35-40. MR 17, 1052.

4. W. Gaschütz, Nichtabelsche p-Gruppen besitzen äussere p-Automorphismen, J. Algebra 4 (1966), 1-2. MR 33 \# 1365.

5. S. A. Jennings, The structure of the group ring of a p-group over a modular field, Trans. Amer. Math. Soc. 50 (1941), 175-185. MR 3, 34.

6. L. E. Moran, The modular group ring of a p-group, M. Phil. Thesis, University of Nottingham, 1972.

7. L. E. Moran and R. Tench, Normal complements in $\bmod p$ envelopes, Pacific J. Math. (to appear).

8. R. Tench, The mod $p$ envelope of a finite $p$-group, M. Sc. Thesis, University of Nottingham, 1973.

DEPARTMENT OF MATHEMatics, University of NotTINGHAM, NotTingham, ENGLAND 